\title{
Comparison of electron drift waves in numerical and analytical tokamak equilibria
}

\author{
T Rafiq, J Anderson and M Persson \\ Department of Electromagnetics and Euratom/VR Association, Chalmers University of \\ Technology, S-41296 Göteborg, Sweden \\ E-mail: elfmp@elmagn.chalmers.se
}

Received 26 March 2003

Published 26 November 2003

Online at stacks.iop.org/PPCF/46/105 (DOI: 10.1088/0741-3335/46/1/007)

\begin{abstract}
In this paper, we demonstrate the importance of the details of the equilibria on the stability of electron drift waves. A comparison of electrostatic electron drift waves in numerical and analytical tokamak equilibria is presented in fully three-dimensional circular and non-circular tokamaks. The numerical equilibria are obtained using the variational moments equilibrium code and the analytical equilibria used is the generalized $\hat{s}-\alpha$ model. An eigenvalue equation for the model is derived using the ballooning mode formalism and solved numerically using a standard shooting technique. The stability and the localization of the electron drift wave is found to be strongly dependent on the local shear of the magnetic field. Large values of the local shear are found to be stabilizing. A disagreement in the results is found between analytical and numerical equilibria at aspect ratios of typical tokamaks, suggesting that the latter approach should be used in the transport calculations. The effects of the local shaping of the magnetic surfaces are complicated and can be both stabilizing and destabilizing, depending on the details of the equilibria.
\end{abstract}

\section{Introduction}

Drift waves are commonly believed to be responsible for the anomalous transport in fusion plasma devices. The ion-temperature-gradient (ITG) driven mode is one of the main candidates for explaining the anomalous transport in the core region [1-7]. The situation at the edge is different, with a strong influence of electron-ion collisions giving a resistive nature to the drift waves in this region.

The effects of plasma shaping on magnetohydrodynamic (MHD) modes are well documented $[8,9]$; however, the effects on drift wave stability are still rather unknown. In transport code simulations it has been suggested that the main effects of plasma shaping on the confinement time originates from the plasma edge region [10]. Nevertheless, there is no consensus on whether the effects of shaping are due to stabilization of the linear modes or due to other indirect effects. The investigation of the influence of plasma shaping is therefore of great interest. 
In this paper, the linear stability of the electron drift waves is examined in analytical and numerical equilibria for circular and JET-like tokamaks. The numerical equilibria are obtained from the three-dimensional equilibrium code variational moments equilibrium code (VMEC) [11]. The generalized $\hat{s}-\alpha$ model is used for analytical equilibria, which allows for variation of the parameters without recomputing the equilibrium [12].

In the model for the electron drift waves, we assume a response close to adiabatic for the electrons and a cold ion response. The reason to consider this simple model is to emphasise the effect of geometry and specifically to examine the role of local magnetic shear and magnetic field curvature on the structure and stability of the modes. The model is formulated in the ballooning representation [13] and the drift wave problem is set as an eigenvalue equation along a magnetic field line. To solve the drift wave equation, we have used the standard shooting technique and are applying Wentzel-Kramers-Brillouin (WKB) type boundary conditions.

In general, we have found that the more unstable modes are most localized. This is true for both circular and non-circular tokamak geometries. However, in a tokamak with non-circular cross-section, modes are found to be more localized than those in a circular equilibrium. The effect of the local shear of the magnetic field is found to be important and that is seen by comparing the circular and the JET-like equilibria. The existence of the high-frequency modes, their localization along the field lines and their frequencies and growth rates are found to be strongly dependent on the local magnetic shear. Moreover, the effect of non-circularity on peaked density profiles is found to be stabilizing for small radii, due to the relatively large local magnetic shear values. However, the effect is quite the opposite at the outer edge region of high $q$-plasmas where local magnetic shear has smaller values.

The remainder of this paper is structured as follows. In section 2, the equilibrium magnetic field is specified in straight field line coordinates and the contravariant and covariant basis vectors are calculated. The equilibrium is computed using the VMEC code with fixed boundary conditions for JET and for a circular tokamak having the same aspect ratio. The generalized $\hat{s}-\alpha$ model is presented in section 3 , which allows a comparison between the circular and non-circular equilibria. In section 4 , the i $\delta$ model is used as a physical model to describe the electron drift modes and is derived from the fluid dynamics for the ions in the electrostatic regime, whereas the electron response is assumed to be close to adiabatic. The resulting equations are closed through the quasi-neutrality condition. In section 5 the results and a discussion thereof are presented. Finally, a summary is given in section 6 .

\section{The magnetic field configuration of the toroidal system}

The magnetic field configurations can be expressed in terms of the Boozer flux coordinates $(s, \theta, \zeta)$, where $\theta$ and $\zeta$ are generalized poloidal and toroidal angles and $s=2 \pi \psi / \psi_{\mathrm{p}}$ is the normalized flux (radial) coordinate [14]. By construction, the radial label ranges from 0 (at the magnetic axis) to 1 (at the last closed magnetic surface). Here, $2 \pi \psi$ is the poloidal magnetic flux bounded by the magnetic axis and the $\psi=$ constant surface and $\psi_{\mathrm{p}}=\pi B_{0} \bar{a}^{2} / q$ the total poloidal magnetic flux. Here, $B_{0}$ is the magnetic field at the axis, $\bar{a}$ is the average minor radius, $q=2 \pi / \iota$ is the safety factor and $\iota=\iota(\psi)$ is the rotational transform that measures the helical twist of the magnetic field lines. The magnetic field, $\boldsymbol{B}$, is expressed as

$$
B=\nabla \alpha \times \nabla \psi=\dot{\psi} \nabla \alpha \times \nabla s, \quad \text { with } \dot{\psi} \equiv \frac{\mathrm{d} \psi}{\mathrm{d} s}=\frac{B_{0} \bar{a}^{2}}{2 q},
$$

which fulfills $\nabla \cdot B=0$ and $B \cdot \nabla \psi=B \cdot \nabla \alpha=0$, implying that $\psi$ and $\alpha$ are stream functions (constant along a field line) of the magnetic field. It further implies that $\psi=$ constant is a magnetic flux surface and that $\alpha=\zeta-q \theta$ is a field line label on this surface. A given magnetic 
flux surface can be represented by a square cell, $0 \leqslant \theta<2 \pi, 0 \leqslant \zeta<2 \pi$, with the edges $\theta=0,2 \pi$ and $\zeta=0,2 \pi$ topologically identified. While the equilibrium is periodic in $\zeta$ and $\theta$, for the eigenvalue problem along the field line, described in the next section, the magnetic field lines lie in the domain $-\infty<\theta<\infty,-\infty<\zeta<\infty$, which is usually referred to as a covering space [15]. This is because in general field lines never close. The equilibrium code VMEC solves the MHD force balance equation,

$$
\boldsymbol{J} \times \boldsymbol{B}=\nabla P,
$$

in three-dimensional geometry. Here, $J=\mu_{0} \nabla \times B$ is the plasma current, $\mu_{0}$ is the permeability of free space and $P$ is the isotropic plasma pressure. In VMEC, two flux surface quantities are prescribed. One is the net toroidal plasma current enclosed within each flux tube and the second is the plasma pressure profile. In this paper we use the plasma pressure profile for all VMEC equilibria. The equilibria for the JET and circular tokamaks are computed with fixed boundary conditions and for a set of 97 magnetic surfaces. The VMEC code uses a coordinate system that has been optimized by minimizing the number of harmonics required to represent the equilibrium quantities so that the equilibrium calculation is very efficient. However, the VMEC coordinate system is not a straight field line coordinate system. Thus, before solving the drift wave equation, we first transform the equilibrium to Boozer coordinates. The VMEC code uses an inverse equilibrium representation, in which the real space cylindrical coordinates are given by Fourier expansions. The Boozer system and the standard cylindrical coordinates $\left(R, \phi_{c}, z\right)$ are related through the Fourier series (for a given magnetic surface, $s=$ constant - a surface that is traced out by a series of magnetic field lines)

$$
\begin{aligned}
& R=\sum_{m=0}^{n_{\mathrm{p}}} \sum_{n=-n_{\mathrm{t}}}^{n_{\mathrm{t}}} R_{m n}(s) \cos (m \theta+n N \zeta), \\
& \phi_{c}=\zeta, \\
& z=\sum_{m=0}^{n_{\mathrm{p}}} \sum_{n=-n_{\mathrm{t}}}^{n_{\mathrm{t}}} z_{m n}(s) \sin (m \theta+n N \zeta) .
\end{aligned}
$$

Here, $n_{\mathrm{p}}$ and $n_{\mathrm{t}}$ are the maximum poloidal and toroidal Fourier components and are both input parameters of VMEC, and $N$ is the number of field period, which is different for different fusion machines. In this case, we use $n_{\mathrm{p}}=47, n_{\mathrm{t}}=1$ and since the tokamak has toroidal symmetry, the field period is 1 . The Fourier coefficients $R_{m n}, \phi_{m n}$ and $z_{m n}$ and the rotational transform, $\iota$, are calculated as functions of the flux coordinate $s$ by the VMEC code [11] and then mapped over to the Boozer coordinate system $(s, \theta, \zeta)[16,17]$. The position vector, $r_{\mathrm{p}}$, of any point $(s, \theta, \zeta)$ at a flux surface $s$ in the coordinate system $(x, y, z)$ is $r_{\mathrm{p}}=\left(R \cos \phi_{c}, R \sin \phi_{c}, z\right)$, where the covariant basis vectors in the Boozer coordinate system are

$$
\boldsymbol{e}_{s}=\frac{\partial \boldsymbol{r}_{\mathrm{p}}}{\partial s}, \quad \boldsymbol{e}_{\theta}=\frac{\partial \boldsymbol{r}_{\mathrm{p}}}{\partial \theta}, \quad \boldsymbol{e}_{\zeta}=\frac{\partial \boldsymbol{r}_{\mathrm{p}}}{\partial \zeta}
$$

and the associated contravariant basis vectors are

$$
\nabla s \equiv \boldsymbol{e}^{s}=\frac{\boldsymbol{e}_{\theta} \times \boldsymbol{e}_{\zeta}}{J}, \quad \nabla \theta \equiv \boldsymbol{e}^{\theta}=\frac{\boldsymbol{e}_{\zeta} \times \boldsymbol{e}_{s}}{J}, \quad \nabla \zeta \equiv \boldsymbol{e}^{\zeta}=\frac{\boldsymbol{e}_{s} \times \boldsymbol{e}_{\theta}}{J} .
$$

The covariant basis vector $e_{\theta}$ is tangent to the $\theta$ coordinate curves, which lie at the intersection of the coordinate surfaces $s=$ constant and $\zeta=$ constant, whereas the contravariant basis vector $\nabla \theta$ is perpendicular to the coordinate surface $\theta=$ constant. The Jacobian (the determinant of the matrix produced by nine partial derivatives) of the transformation $J$ can be calculated using the set of covariant basis vectors

$$
J \equiv \boldsymbol{e}_{s} \cdot \boldsymbol{e}_{\theta} \times \boldsymbol{e}_{\zeta}=\frac{\dot{\psi}}{B^{2}}\left(B_{\theta}+q B_{\zeta}\right),
$$


where $B_{\theta}$ and $B_{\zeta}$, the covariant components of $B$, are surface quantities, i.e. $B_{\theta}=B_{\theta}(s)$ and $B_{\zeta}=B_{\zeta}(s)$. Having calculated the derivatives of the cylindrical coordinates $R, \phi_{c}$ and $z$ with respect to $s$ numerically, equation (6) can be used to calculate derivatives of $B$ with respect to $\theta$ and $\zeta$ analytically, while the derivatives with respect to $s$ are calculated numerically. Using the reciprocal relation of contravariant and covariant basis vectors, equation (1) can be written as $B=\dot{\psi} / J\left(\boldsymbol{e}_{\theta}+q \boldsymbol{e}_{\zeta}\right)$, and the parallel gradient operator, $e_{\|} \cdot \nabla$ (where $e_{\|}=B / B$ ), can be written as

$$
\boldsymbol{e}_{\|} \cdot \nabla=\frac{\dot{\psi}}{J B}\left(\frac{\partial}{\partial \theta}+q \frac{\partial}{\partial \zeta}\right)=\left.\frac{\dot{\psi} q}{J B} \frac{\mathrm{d}}{\mathrm{d} \zeta}\right|_{\text {field line }}
$$

and the field line curvature vector, $\kappa$, is

$$
\boldsymbol{\kappa} \equiv \boldsymbol{e}_{\|} \cdot \nabla \boldsymbol{e}_{\|}=q\left(\frac{\dot{\psi}}{J B}\right)^{2}\left[\frac{\mathrm{d}}{\mathrm{d} \zeta}\left(\boldsymbol{e}_{\theta}+q \boldsymbol{e}_{\zeta}\right)-\frac{1}{2} \frac{\mathrm{d} \ln J}{\mathrm{~d} \zeta}\left(\boldsymbol{e}_{\theta}+q \boldsymbol{e}_{\zeta}\right)\right] .
$$

When the curve under consideration lies within a surface, it is customary to define curvature components with respect to the surface. The component of $\kappa$ normal to the surface is called the normal curvature, $\kappa_{\mathrm{n}}$, whereas the component of $\kappa$ tangent to the surface is referred to as the geodesic curvature, $\kappa_{\mathrm{g}}$. The normal and geodesic components of $\kappa$ are

$$
\kappa_{\mathrm{n}}=\kappa \cdot \frac{\nabla s}{|\nabla s|}, \quad \kappa_{\mathrm{g}}=\kappa \cdot\left(\frac{\nabla s}{|\nabla s|} \times \boldsymbol{e}_{\|}\right) .
$$

Regions of the configurations with a negative normal curvature are expected to be unstable to local pressure-driven instabilities, whereas positive regions are expected to be stable. The design of the magnetic confinement devices are often numerically optimized to enhance the positive average curvature.

Using equation (6), we write

$\nabla \ln B=\frac{1}{2}\left[\left(\frac{\mathrm{d}}{\mathrm{d} s} \ln \left(B_{\theta}+q B_{\zeta}\right)-\frac{\partial \ln J}{\partial s}\right) \nabla s-\frac{\partial \ln J}{\partial \theta} \nabla \theta-\frac{\partial \ln J}{\partial \zeta} \nabla \zeta\right]$

and

$$
\frac{\partial J}{\partial s}=\frac{\partial \boldsymbol{e}_{s}}{\partial s} \cdot \boldsymbol{e}_{\theta} \times \boldsymbol{e}_{\zeta}+\boldsymbol{e}_{s} \cdot \frac{\partial \boldsymbol{e}_{\theta}}{\partial s} \times \boldsymbol{e}_{\zeta}+\boldsymbol{e}_{s} \cdot \boldsymbol{e}_{\theta} \times \frac{\partial \boldsymbol{e}_{\zeta}}{\partial s} .
$$

The local magnetic shear is the local rate of rotation of the magnetic field direction and is another equilibrium quantity that plays an important role in the stability [18] and can be written as [19]

$$
S=\frac{\dot{\psi}}{B}\left[\frac{\nabla \alpha \cdot \nabla s}{|\nabla s|}\left(\frac{\nabla s}{|\nabla s|} \cdot \nabla\right) \nabla s-(\nabla s \cdot \nabla) \nabla \alpha-(\nabla \alpha \cdot \nabla) \nabla s\right] \cdot e_{\|} \cdot
$$

\section{The magnetic field configuration in the $\hat{s}-\alpha$ model}

As a model for an analytical equilibrium we employ the generalized $\hat{s}-\alpha$ model of [12], which allows for modification of the equilibrium parameters like elongation and Shafranov shift without recomputing the equilibrium. The flux coordinate system is defined by

$$
\begin{aligned}
& R(r, \theta)=\sum_{n=0}^{\infty} R_{n}(r) \cos (n \theta), \\
& Z(r, \theta)=\sum_{n=0}^{\infty} Z_{n}(r) \sin (n \theta),
\end{aligned}
$$


where $R$ and $Z$ are the usual cylindrical coordinates. The terms $R_{0}, R_{1}=r, Z_{1}=r \kappa$ describe the major radius, minor radius and ellipticity, respectively, and the terms $R_{2}, Z_{2}$ describe triangularity. The scale factors become

$$
\begin{aligned}
& g_{r r}=\left(\partial_{r} R_{0}+\cos \theta\right)^{2}+\left(\partial_{r}(r \kappa) \sin \theta\right)^{2}, \\
& g_{\theta \theta}=r^{2}\left(1+\left(\kappa^{2}-1\right) \cos ^{2} \theta\right), \\
& g_{r \theta}=\partial_{r}(\kappa r) \kappa r \sin \theta \cos \theta-r\left(\partial_{r} R_{0}+\cos \theta\right) \sin \theta, \\
& g_{\phi \phi}=R^{2},
\end{aligned}
$$

where $\kappa$ is the elongation and $\partial_{j}=\left(\partial / \partial x^{j}\right)$ (note that it only acts on the object directly following), and we denote the position vector $\vec{r}=(R, Z)$ and the basis vectors $\hat{e}_{j}=1 / h_{j} \partial_{j} \vec{r}$. The metric tensor, $g_{i j}$, is defined as

$$
\mathrm{d} s^{2}=g_{i j} \mathrm{~d} x^{i} \mathrm{~d} x^{j} .
$$

Keeping only the first terms in inverse aspect ratio $\epsilon=a / R$-ordering, where $a$ and $R$ are the minor and major radius of the plasma, respectively, and with the $B$-field given by $\vec{B}=B_{\theta} \hat{\theta}+B_{\phi} \hat{\phi}$, we have the magnetic drift frequency and perpendicular wave vector, $k_{\perp}$, as

$$
\begin{gathered}
\frac{\omega_{\mathrm{D}}}{\omega_{* e}}=\epsilon_{\mathrm{n}} g(\theta) \approx \frac{\epsilon_{\mathrm{n}}}{\sqrt{g_{\phi \phi}}}\left(g^{\theta r} \Gamma_{\phi \phi}^{r} J \frac{q}{r} s+g^{\theta \theta} \Gamma_{\phi \phi}^{r} J q-g^{r r} \Gamma_{\phi \phi}^{\theta} J \frac{q}{r} s-g^{\theta r} \Gamma_{\phi \phi}^{\theta} J q\right), \\
k_{\perp}^{2}=k_{\theta}^{2} k(\theta) \approx-\frac{k_{\theta}^{2}}{J^{2}}\left(g_{r r}+g_{\theta \theta}(s \theta)^{2}+2 g_{r \theta} s \theta\right),
\end{gathered}
$$

where $\hat{s}=\mathrm{d} \ln q / \mathrm{d} \ln r$ is the global magnetic shear. Here, the inverse of the metric tensor is defined by the relation $g_{i j} g^{j k}=\delta_{i}^{k}$. The Christoffel symbols are given by

$$
\begin{aligned}
& \Gamma_{i k}^{j}=\frac{1}{2} g^{j \mathrm{n}}\left(\partial_{k} g_{\mathrm{n} i}+\partial_{i} g_{\mathrm{n} k}-\partial_{j} g_{i k}\right), \\
& \Gamma_{\phi \phi}^{r}=-\frac{1}{4}\left(\frac{g_{\theta \theta}}{J^{2}} \partial_{r} g_{\phi \phi}^{2}-\frac{g_{r \theta}}{J^{2}} \partial_{\theta} g_{\phi \phi}^{2}\right), \\
& \Gamma_{\phi \phi}^{\theta}=-\frac{1}{4}\left(-\frac{g_{\theta r}}{J^{2}} \partial_{r} g_{\phi \phi}^{2}+\frac{g_{r r}}{J^{2}} \partial_{\theta} g_{\phi \phi}^{2}\right),
\end{aligned}
$$

where the Jacobian is given by

$$
J^{2}=g_{\phi \phi}\left(g_{r r} g_{\theta \theta}-g_{r \theta}^{2}\right) .
$$

These results reduce to the usual expressions in circular geometry, i.e.

$$
\begin{aligned}
& \frac{\omega_{\mathrm{D}}}{\omega_{* e}} \approx \epsilon_{\mathrm{n}}(\cos \theta+s \theta \sin \theta), \\
& k_{\perp}^{2} \approx k_{\theta}^{2}\left(1+s^{2} \theta^{2}\right) .
\end{aligned}
$$

The effects of triangularity are neglected in the analytical equilibrium. A previous study by Hua et al [20], using a high- $n$ linear ballooning mode code for ITG modes, showed that the growth rates in the plasma core decrease with elongation, but are insensitive to triangularity. Meanwhile, Waltz and Miller [6], using a non-linear ballooning mode gyrofluid code for ITG turbulence, showed that the growth rate is only slightly modified for small values of triangularity. However, high and reversed triangularity give improvements. Furthermore, Belli et al [21] used a gyrokinetic code and found that increasing the triangularity and triangularity gradient at a finite elongation has a stabilizing influence at high $\alpha$ (here $\alpha \approx-R_{0} / q^{2} \partial \beta / \partial \rho$ ), but at high shear, however, a high degree of shaping effects is found to have a destabilizing influence at low $\alpha$. The numerical equilibria we use here have a low $\beta$ and small triangularity ( 0.11 ) on the magnetic surface $s=0.75$. Therefore, due to a small triangularity and low $\beta$, we expect weak effects on the growth rate, and hence triangularity is neglected in the analytical equilibrium, whereas it is included in the numerical equilibrium. 


\section{The drift wave model}

In the three-dimensional geometry described before, we use a simple drift wave model in which ions are treated as a cold magnetized fluid and the electrons are assumed to be close to adiabatic. The eigenmode equation is then derived from the quasi-neutrality condition. The plasma is assumed to be in the collisional regime so that particle trapping and detrapping effects are not important. A ballooning representation is used for the electrostatic potential perturbation, $\hat{\phi}=\Phi(\zeta) \exp \left[\mathrm{i} \epsilon^{-1} S(\psi, \alpha)\right]$, where $\epsilon$ is the WKB expansion parameter, $S$ is the eikonal and $\Phi$ is the envelope [22]. The wave equation can be written as

$$
\frac{\bar{R}^{2}}{\Omega^{2}} \nabla \cdot \nabla_{\|} \hat{\phi}-\left[\mathrm{i} \frac{A}{\Omega}\left(p_{*}+p_{\mathrm{d}}\right) \cdot \nabla_{\perp}+\frac{A^{2}}{\bar{R}^{2} B^{2}} \nabla_{\perp}^{2}-1-\mathrm{i} \delta\right] \hat{\phi}=0,
$$

where $A=\bar{R} B_{0} \rho_{s}, \bar{R}$ is the average major radius, $B_{0}$ is the magnetic field at the axis, $\rho_{s 0}=c_{s} / \omega_{\mathrm{ci} 0}, c_{s}=\sqrt{\left(T_{\mathrm{e}} / m_{\mathrm{i}}\right)}$ is the ion thermal velocity evaluated at the electron temperature and $\omega_{\mathrm{ci} 0}$ is the ion cyclotron frequency evaluated at the magnetic axis. The frequency is normalized with respect to $c_{s} / \bar{R}\left(\Omega=\bar{R} \omega / c_{s}\right)$ and $\hat{\phi} \equiv e \phi / T_{\mathrm{e}}$ is the normalized perturbed potential. The parameters $\boldsymbol{p}_{*}$ and $\boldsymbol{p}_{\mathrm{d}}$ are proportional to the diamagnetic and the magnetic drift velocities and we have defined

$$
\boldsymbol{p}_{*}=-\frac{\boldsymbol{B} \times \nabla \ln n}{B^{2}}, \quad \boldsymbol{p}_{\mathrm{d}}=\frac{\boldsymbol{B} \times(\boldsymbol{\kappa}+\nabla \ln B)}{B^{2}}
$$

and $\delta$ represents the non-adiabatic response of the electrons and can be due to collisions or any other dissipative mechanism. In deriving the above equation we also assume that the characteristic perpendicular wavelength is much larger than the ion thermal gyro-radius.

By analogy with the Fourier form, the perpendicular wavenumber is

$$
\begin{aligned}
\boldsymbol{k}_{\perp} & =\mathrm{i} \nabla_{\perp} \ln \hat{\phi}=\epsilon^{-1} \nabla_{\perp} S=\frac{1}{\epsilon} \frac{\partial S}{\partial \alpha}\left\{\nabla \alpha+\frac{\partial S / \partial \psi}{\partial S / \partial \alpha} \nabla \psi\right\} \\
& =\epsilon^{-1} \frac{\partial S}{\partial \alpha}\left[\nabla \zeta-q \nabla \theta-\left\{\theta \frac{\mathrm{d} q}{\mathrm{~d} \psi}-\frac{\partial S / \partial \psi}{\partial S / \partial \alpha}\right\} \nabla \psi\right] .
\end{aligned}
$$

Equation (26) is to be solved along a given field line, labelled by $\alpha$, on a flux surface $\psi$. This field line passes through the point $\left(\theta_{0}, \zeta_{0}\right)$. The equilibrium is periodic and therefore invariant under the operations

$\alpha\left(\psi, \theta_{0}, \zeta_{0}\right) \rightarrow \alpha\left(\psi, \theta_{0}+2 \pi, \zeta_{0}\right) \quad$ and $\quad \alpha\left(\psi, \theta_{0}, \zeta_{0}\right) \rightarrow \alpha\left(\psi, \theta_{0}, \zeta_{0}+2 \pi\right)$

We want $\boldsymbol{k}_{\perp}$ to fulfil the same periodicity. This is obtained if we require that

$$
\frac{\partial S / \partial \psi}{\partial S / \partial \alpha}=\left(\theta_{0}+\theta_{k}\right) \frac{\mathrm{d} q}{\mathrm{~d} \psi}
$$

Note that the definition of $\theta_{k}$ differs slightly from that used in [15]. Here, a fixed $\theta_{k}$ gives a constant local value of the normal component of the perpendicular wave number as $\theta_{0}$ and $\zeta_{0}$ are varied. To see this we use the field line equation

$$
\zeta-q \theta=\alpha=\zeta_{0}-q \theta_{0}
$$

and write equation (27) in the form

$$
\boldsymbol{k}_{\perp}=\epsilon^{-1} \frac{\partial S}{\partial \alpha}\left[\nabla \zeta-q \nabla \theta-\left(\frac{\zeta-\zeta_{0}}{q}-\theta_{k}\right) \frac{\mathrm{d} q}{\mathrm{~d} \psi} \nabla \psi\right] .
$$

From this expression it is clear we are looking at the same mode as we make calculations at various matching points $\theta_{0}, \zeta_{0}$ on the magnetic surface. 
Writing $\nabla_{\|}=e_{\|}\left(e_{\|} \cdot \nabla\right)$ and employing equation (7), the operator $\nabla \cdot \nabla_{\|}$can be written in terms of the field line parameter $\zeta$ as

$$
\nabla \cdot \nabla_{\|}=\left.\left(\frac{\dot{\psi} q}{J B}\right)^{2} \frac{\mathrm{d}^{2}}{\mathrm{~d} \zeta^{2}}\right|_{\text {field line }}
$$

Then, the drift wave equation, equation (26), may be written in the form of a Schrödingerlike equation,

$$
\frac{\mathrm{d}^{2} \Phi}{\mathrm{d} \zeta^{2}}=U \Phi
$$

where the potential, $U$, is

$$
U(\zeta, \Omega)=\left(\frac{J B}{q \bar{R} \dot{\psi}}\right)^{2}\left\{\left(\Omega_{*}+\Omega_{\mathrm{d}}\right) \chi \Omega-\left(1+\frac{B_{0}^{2} \chi^{2}\left|\hat{\boldsymbol{k}}_{\perp}\right|^{2}}{B^{2}}+\mathrm{i} \delta\right) \Omega^{2}\right\}
$$

and where

$$
\begin{gathered}
\Omega_{*}=\Omega_{*}(s)=-\frac{2 \bar{R}}{L_{\mathrm{n}}}, \quad L_{\mathrm{n}}^{-1}=-\frac{\mathrm{d} \ln n_{0}}{\bar{a} \mathrm{~d} s}, \quad \chi=\epsilon^{-1} \frac{q \rho_{s 0}}{\bar{a}} \frac{\partial S}{\partial \alpha}, \\
\Omega_{\mathrm{d}}=\Omega_{\mathrm{d}}(s, \alpha, \zeta)=B_{0} \bar{R} \boldsymbol{p}_{\mathrm{d}} \cdot \hat{\boldsymbol{k}}_{\perp}, \\
\hat{\boldsymbol{k}}_{\perp}=\frac{\bar{a}}{q}\left[\nabla \zeta-q \nabla \theta-\left(\theta-\theta_{0}-\theta_{k}\right) \dot{q} \nabla s\right], \quad \theta=\frac{\alpha-\zeta}{q}, \quad \theta_{0}=\frac{\alpha-\zeta_{0}}{q} .
\end{gathered}
$$

The physical problem is now reduced to an eigenvalue equation along the field line, which is similar to the problem of a particle in a potential well in quantum mechanics. Hence, equation (28) is solved numerically along a magnetic field line $(\alpha)$ on a given flux surface $(s)$. This is solved by applying an appropriate boundary condition for a large $|\zeta|$ and by demanding continuity of the eigenfunction and its first derivative at a point $\zeta=\zeta_{0}$, usually referred to as a 'matching point'. Hence, at a given magnetic surface $s$, the eigenfunction $\Phi$ and the corresponding normalized eigenfrequency, $\Omega$, can be determined for given values of the equilibrium plasma density scale length, $L_{\mathrm{n}}$, and the free parameters $\chi$ and $\theta_{k}$. The former controls the magnitude of the perpendicular wave vector, $k_{\perp}$, and the latter its orientation.

\section{Numerical results and discussion}

The drift wave equation is solved by a shooting algorithm using a sixth-order Numerov scheme and WKB-type boundary conditions and by demanding continuity of the function at its first derivative at a matching point. Details of the boundary conditions and the numerical method used are given in [23].

The input pressure profile used in the calculation of the magnetic field configuration is shown in figure 1 together with the $q$-profile, which is an output from the code. The poloidal cross section of the non-circular tokamaks is provided in figure 2 (right). For the sake of clarity, only 19 of the nested magnetic surfaces are plotted. Analytical circular and non-circular crosssections used in comparison with to the numerical equilibria are shown in figure 2 (left). The full equilibrium has been determined with 97 magnetic surfaces. The average major radius $(\bar{R})$ is $2.96 \mathrm{~m}$, the magnetic field strength at the magnetic axis is $3.45 \mathrm{~T}$ and the aspect ratio is $\bar{R} / \bar{a}=1.77$ ( $\bar{a}$ is the average minor radius).

Figure 3 shows the drift wave spectrum calculated on the flux surface $s=0.65$ using the matching point $\left(\theta_{0}=0, \zeta_{0}=0\right)$. Here the local magnetic shear is small, normal curvature is large negative (unfavourable) and geodesic curvature is zero. The parameter 

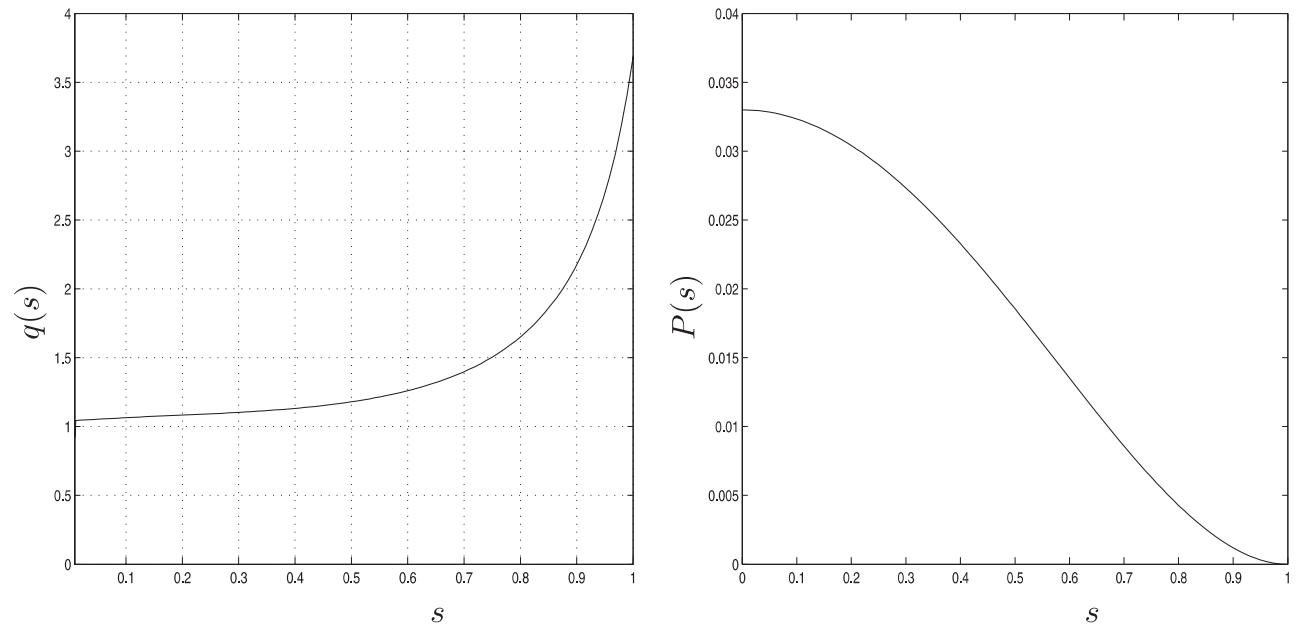

Figure 1. The output $q$ profile (left) and input pressure profile (right) used in the calculation of the magnetic field configuration.
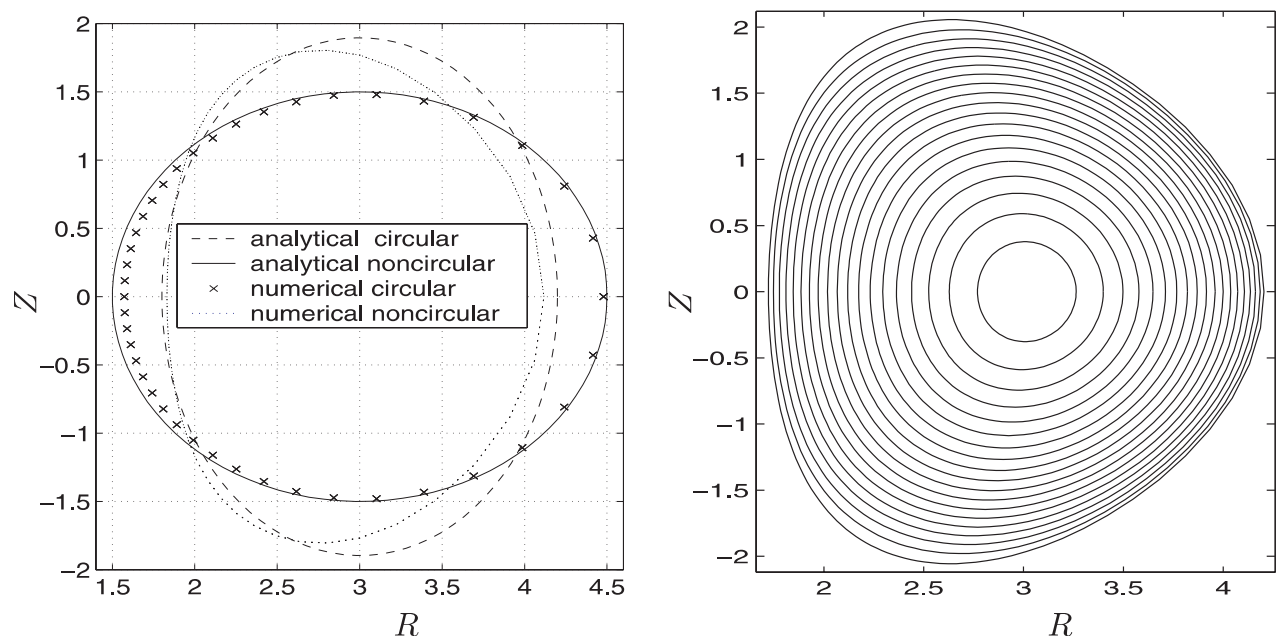

Figure 2. The analytical and the numerical equilibrium cross sections (left) used as a comparison and the reduced non-circular magnetic flux surfaces (right).

values used are $b=\left(k_{\perp} \rho_{s}\right)^{2}=\chi^{2}\left|\hat{k}_{\perp} \cdot \hat{k}_{\perp}\right|_{\zeta=\zeta_{0}}=\left(\epsilon^{-1} \partial s / \partial \alpha\right)^{2}|\nabla \alpha \cdot \nabla \alpha|_{\zeta=\zeta_{0}}=0.1$ and $\theta_{k}=0, \epsilon_{\mathrm{n}}=L_{\mathrm{n}} / \bar{R}=0.05$, where $L_{\mathrm{n}}$ is the radial density scale length, and $L_{\mathrm{n}}^{-1}=-\mathrm{d} \ln n_{0} /\left.\mathrm{d} s \hat{s} \cdot \nabla s\right|_{\zeta=\zeta_{0}}$, where $\hat{s} \equiv \nabla s /|\nabla s|$ is a unit vector normal to the magnetic surface and pointing outwards. In the case of an axisymmetric circular tokamak

$$
\psi=B_{0} \int \frac{r}{q} \mathrm{~d} r, \quad \nabla s=\hat{r} \frac{2 q(\bar{a}) r}{\bar{a}^{2} q}, \quad \dot{\psi}=\frac{B_{0} \bar{a}^{2}}{2 q(\bar{a})},
$$

therefore $s$ relates to $r$ as

$$
s=\frac{2 q}{\bar{a}^{2}} \int \frac{r}{q(r)} \mathrm{d} r, \quad \nabla s=\frac{2 q r}{\bar{a}^{2} q(r)} \nabla r
$$




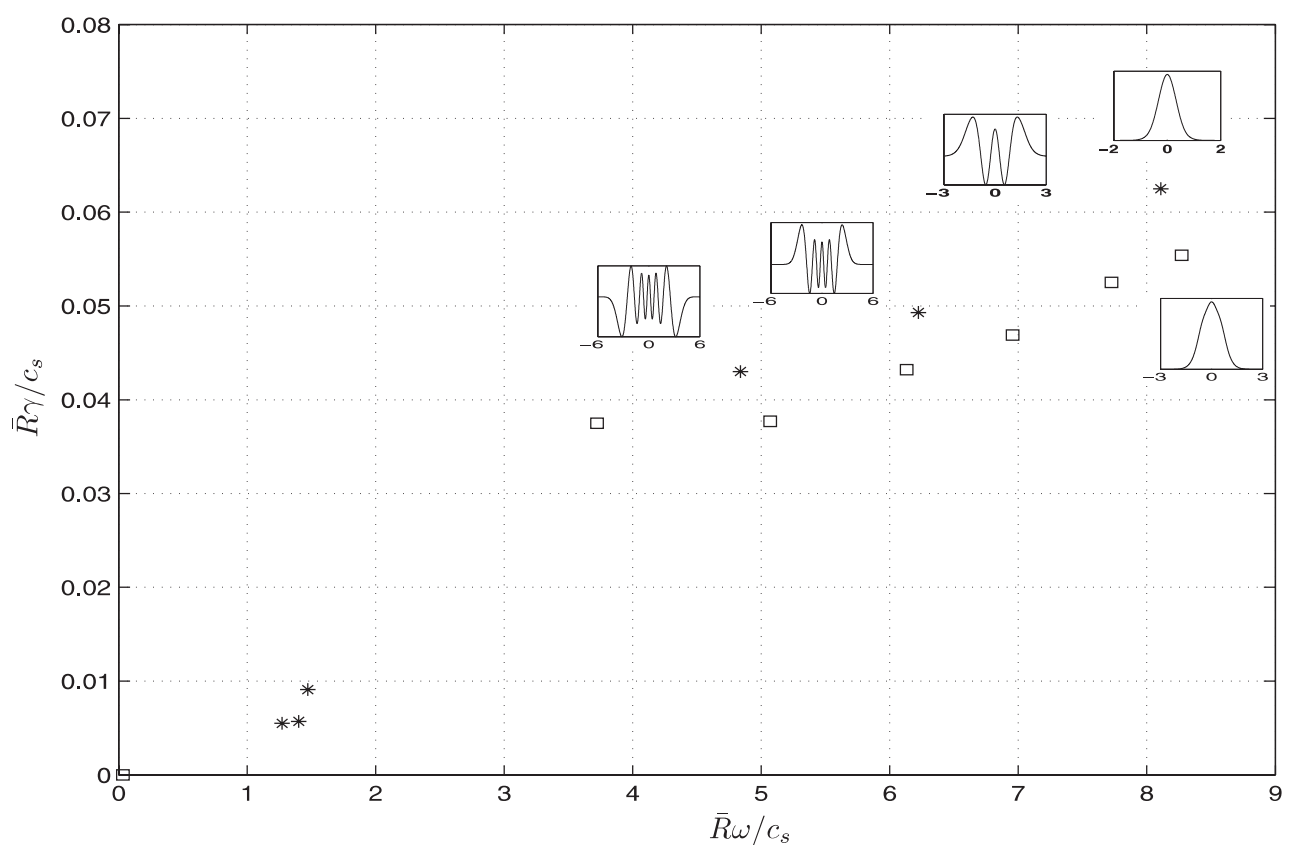

Figure 3. The drift wave spectrum (the growth rate, $\bar{R} \gamma / c_{s}$, versus normalized real frequency, $\bar{R} \omega / c_{s}$ ) obtained for $\epsilon_{\mathrm{n}}=0.05, b=0.1, \theta_{k}=0, \delta=0.01$, along the field line passing through $\theta_{0}=0, \zeta_{0}=0$ on the flux surface $s=0.65$. Asterisks represent the non-circular tokamak, and squares the circular tokamak.

and density gradient scale length in terms of $r$ can be written as

$$
L_{\mathrm{n}}^{-1}=-\left.\frac{\mathrm{d} \ln n_{0}}{\mathrm{~d} s} \hat{s} \cdot \nabla s\right|_{\zeta=\zeta_{0}}=-\frac{\mathrm{d} \ln n_{0}}{\mathrm{~d} r}|\nabla r|_{\zeta=0} .
$$

Eigenfunctions in different parts of the spectrum are shown as insets. At low frequencies the modes are extended along the field line and for higher frequencies they are more localized. This is the case for both circular and non-circular tokamaks. For the non-circular case, the growth rate is somewhat larger than for the circular configuration, whereas the real frequency is found to be similar. However, the envelope of the eigenfunction in the non-circular case is found to be more localized than the circular case. Both the larger value of the growth rate and the localization of the modes along the field line can be understood by examining the local characteristics of the field.

Figure 4 shows the eigenfunctions of the most unstable modes, which are plotted as a function of generalized toroidal angle, $\zeta$; local magnetic shear, $|\boldsymbol{B}|$, and normal curvature for circular and non-circular tokamaks are also superimposed. In both cases, the most unstable mode is found to be localized in unfavourable curvature, in the minimum of $|\boldsymbol{B}|$ and in the minimum of the local magnetic shear. However, increasing the local magnetic shear has a major influence on the favourable behaviour of the circular configuration over the non-circular configuration on this specific field line. Furthermore, the role of the local magnetic shear in localizing and constraining the mode along the field line is also seen. The driving effect of the normal component of the curvature is found to be almost the same in both configurations.

Figure 5 shows the variation of the magnitude of the local magnetic shear, $S$, and $\hat{k}_{\perp}^{2}$ as a function of the ballooning coordinate (non-periodic), $\zeta$, on the magnetic surface $s=0.75$ along the magnetic field line specified $\alpha=0$. This field line contains $\theta_{0}=0, \zeta_{0}=0$. The 

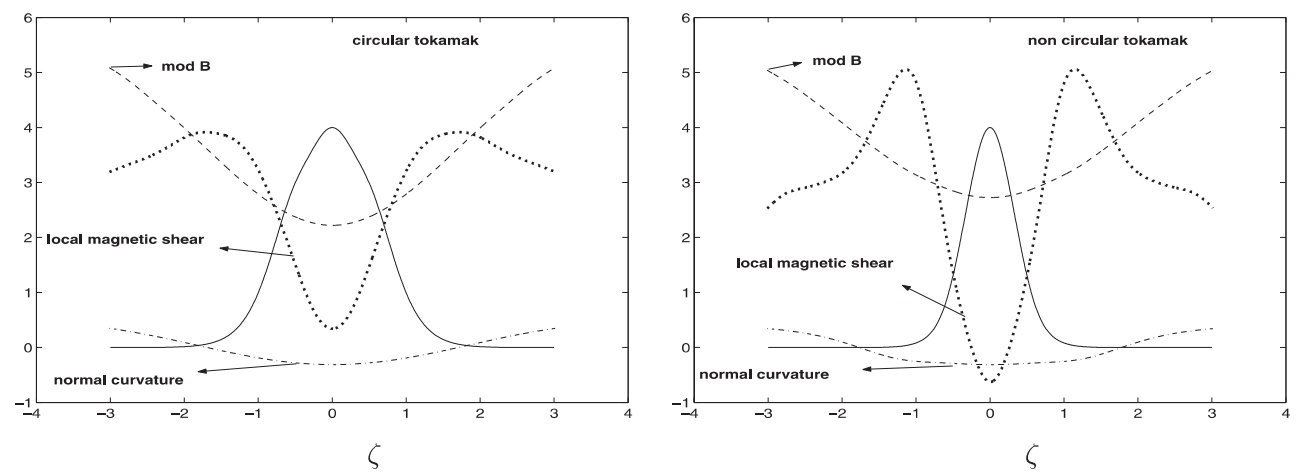

Figure 4. Eigenfunction (left for circular and right for non-circular tokamak) of most unstable mode as a function of $\zeta$ for the case of figure 1. The strength of the field line $|\boldsymbol{B}|$ (- - - -), normal curvature $\kappa_{\mathrm{n}}(-\cdot-)$ and local magnetic shear $(\cdots \cdots)$ are also plotted.
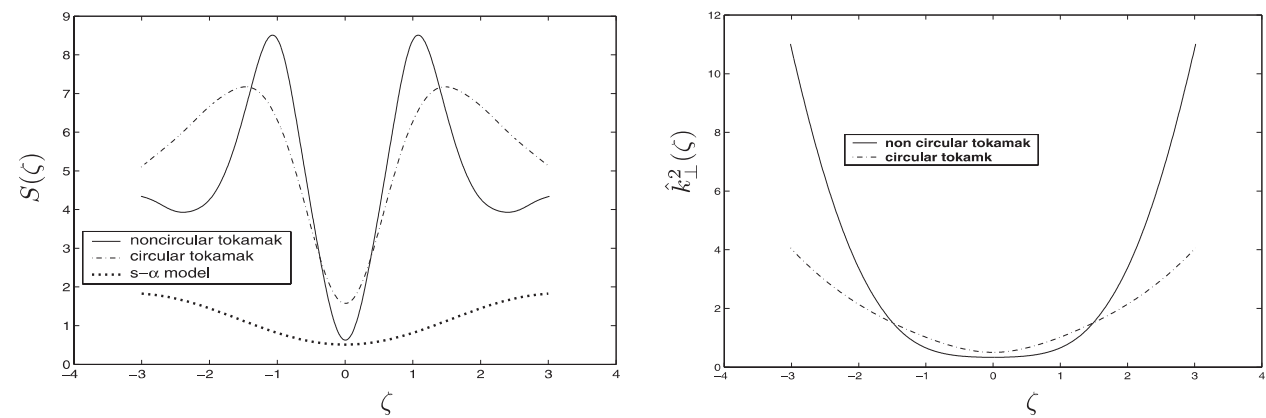

Figure 5. Variation of $S(\zeta)$ (left) and $\hat{k}_{\perp}^{2}(\zeta)$ (right) along a magnetic field line with $\theta_{0}=0$, $\zeta_{0}=0$ on the magnetic surface $s=0.75$. The dash-dotted line represents the circular cross section tokamak, dotted line the simple circular infinite aspect ratio $\hat{s}-\alpha$ model equilibrium and solid line the JET.
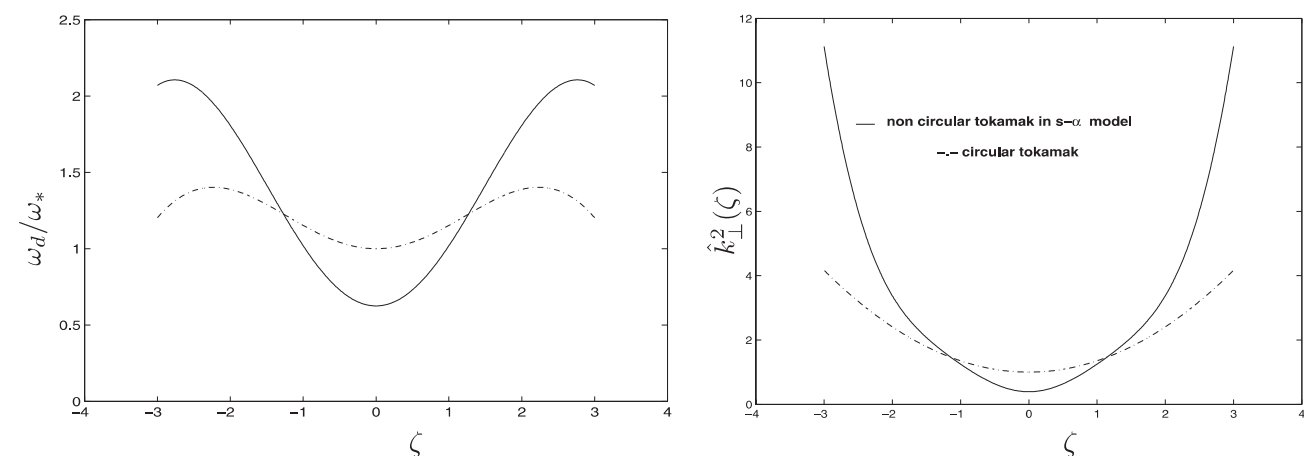

Figure 6. Variation of $\omega_{\mathrm{d}} / \omega_{*}(\zeta)$ (left) and $\hat{k}_{\perp}^{2}(\zeta)$ (right) using analytical equilibria. The dashed line represents the circular cross section tokamak and solid line the non-circular $(\kappa=1.58)$ tokamak.

solid line represents a non-circular tokamak and the dash-dotted line is for a circular tokamak. A higher value of local magnetic shear is found for the circular case near $\zeta=0$ than for the non-circular case. Therefore, in the circular case, a relatively lower growth rate is expected for highly localized modes along the field line. Figure 6 shows the variation of $\omega_{\mathrm{d}} / \omega_{*}(\zeta)$ 

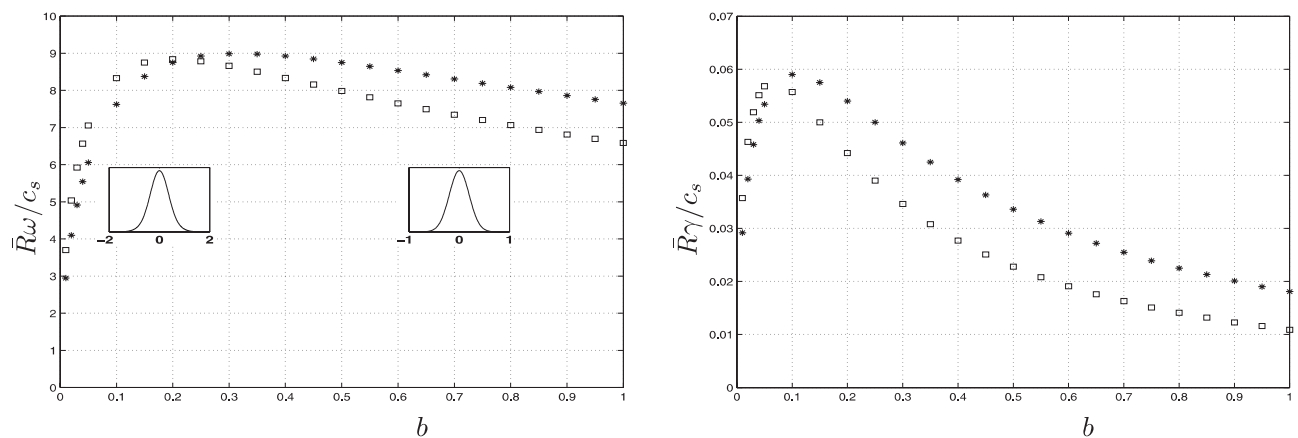

Figure 7. Normalized real frequency, $\bar{R} \omega / c_{s}$ (left), and growth rate, $\bar{R} \gamma / c_{s}$ (right), as a function of $b$ (asterisks representing the JET-like equilibrium and squares the circular equilibrium) for $\epsilon_{\mathrm{n}}=0.05, \theta_{k}=0, \alpha=0$ and $s=0.75$.
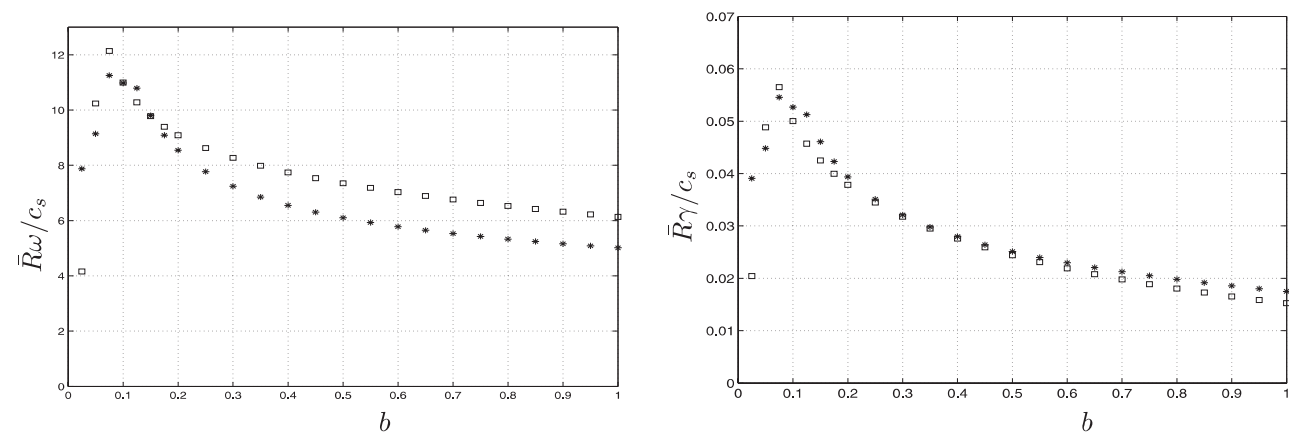

Figure 8. Normalized real frequency, $\bar{R} \omega / c_{s}$ (left), and growth rate, $\bar{R} \gamma / c_{s}$ (right), as a function of $b$ (asterisks representing the elongated $(\kappa=1.58)$ and squares the circular $(\kappa=1.0)$ analytical equilibria); other parameters are the same as in figure 7.

and $\hat{k}_{\perp}^{2}(\zeta)$ using analytical equilibria for $q=1.5$ and $\kappa=1.58$ for the non-circular case and $\kappa=1.0$ for the circular case. A good agreement between the analytical and the numerical results is found in the case of the perpendicular wave vector.

Figure 7 gives the mode eigenfrequency (both real frequency and growth rate) of the most unstable modes as a function of $b$ on a magnetic surface $s=0.75$, and other parameters are the same as in figure 3. The asterisks represent the non-circular tokamak and the squares the circular tokamak. It is found that as $b$ increases the growth rate and the real frequencies of the modes decrease, which is consistent with earlier tokamak studies. The difference between the two cases is that the maximum growth rate occurs at $b=0.1$ for the non-circular case, while it occurs for $b<0.1$ for the circular case. This shift towards a shorter wavelength for elongated equilibria tends to reduce the transport by reducing the correlation length in the plasma. However, while a stabilization effect is found in the circular case for higher values of $b$ (shorter wavelengths), at longer wavelengths the mode growth decreases more rapidly for the non-circular case. As can be seen from the inset eigenfunctions, the modes become more localized along the field line for shorter wavelengths, and feel the higher values of the local magnetic shear, as earlier discussed in connection with figure 5 .

Figure 8 shows the same results for the analytical equilibria. The asterisks represent the non-circular tokamak and the squares the circular tokamak. The results of numerical and analytical equilibria with respect to plasma shaping are qualitatively similar. For the 

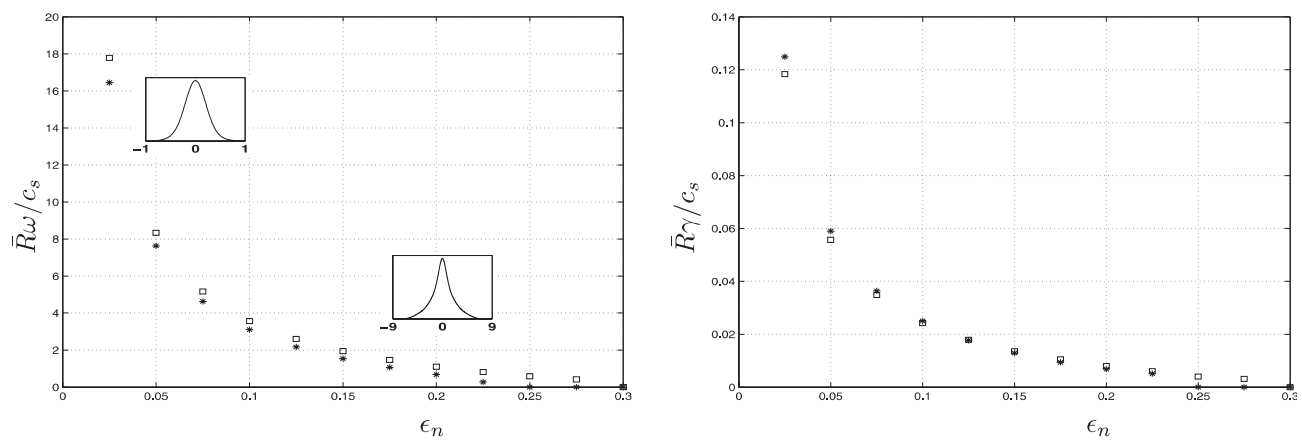

Figure 9. Normalized real frequency, $\bar{R} \omega / c_{s}$ (left), and growth rate, $\bar{R} \gamma / c_{s}$ (right), as a function of $\epsilon_{\mathrm{n}}$ (asterisks representing the JET and squares the circular equilibria) for $b=0.1$; other parameters are the same as in figure 7.
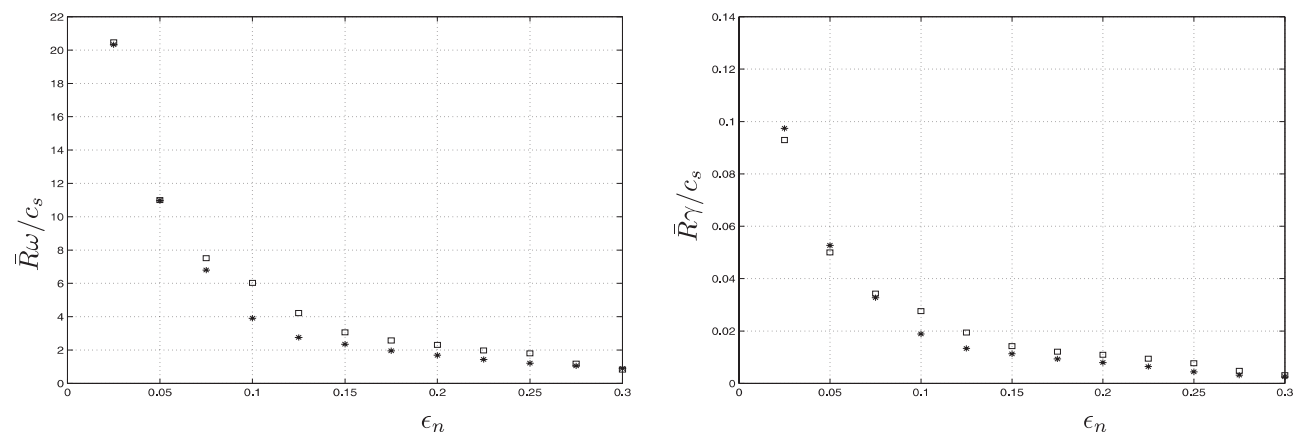

Figure 10. Normalized real frequency, $\bar{R} \omega / c_{s}$ (left), and growth rate, $\bar{R} \gamma / c_{s}$ (right), as a function of $\epsilon_{\mathrm{n}}$ (asterisks representing the elongated $(\kappa=1.58)$ and squares the circular $(\kappa=1.0)$ analytical equilibria) for $b=0.1$; other parameters are the same as in figure 8 .

real frequencies there is a shift towards smaller values for the analytical equilibria where the largest differences are found for the non-circular tokamak. For the growth rates the situation is reversed, with the best agreement found in the non-circular case. Note that for larger values of $b$ the numerical growth rates in circular and non-circular tokamaks differ by more than $50 \%$ but not at all for the analytical case.

In figure 9 the growth rates and the real frequencies are presented as functions of $\epsilon_{\mathrm{n}}$, and otherwise using the same parameter values as in figure 7. In both cases, the mode is stabilized by compressional effects for large $\epsilon_{\mathrm{n}}$. This happens in the flat density regime, $\epsilon_{\mathrm{n}}>0.25$, which extends over a large portion of the plasma radius in tokamaks [24]. We also note that for large $\epsilon_{\mathrm{n}}$ the stable regime appears earlier for the non-circular case than for a circular tokamak. However, the effects of elongation are slightly destabilizing for peaked density profiles $\left(\epsilon_{\mathrm{n}}<0.1\right)$, typical for an edge plasma discharge. Therefore, the mode is expected to be more strongly destabilized at the outer part of the discharge. This is due to the fact that the local magnetic shear in the non-circular case is slightly smaller than the circular case in the region of the mode localization, as can be seen from figure 5. The same $\epsilon_{\mathrm{n}}$ function is plotted in figure 10 for analytical equilibria. We note that for the numerical and analytical equilibria the results are qualitatively similar.

The parameter $\theta_{k}$ controls the component of the wave vector along the normal of the flux surface and appears in the wave equation through $\hat{k}_{\perp}$. When $\theta_{k}$ is increased, the wave vector 

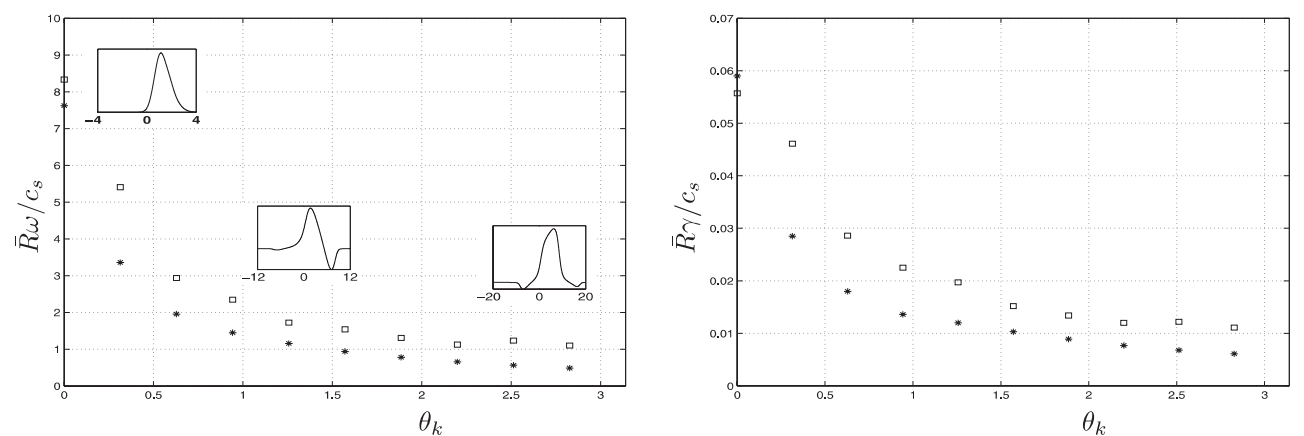

Figure 11. Normalized real frequency, $\bar{R} \omega / c_{s}$ (left), and growth rate, $\bar{R} \gamma / c_{s}$ (right), as a function of $\theta_{k}$ (asterisks represent the JET and squares the circular equilibria) for $\epsilon_{\mathrm{n}}=0.05, b=0.1, \alpha=0$ and $s=0.75$.

rotates in the plane perpendicular to $\boldsymbol{B}$. This is also a free parameter and so the spectrum should be scanned over this parameter. Note that our $\theta_{k}$ is not periodic and differs slightly from the $\theta_{k}$ used in [15]. Our $\theta_{k}$ is chosen so as to keep the orientation of the wave vector at the matching point normal to the magnetic flux surface zero for all the points $\left(\theta_{0}, \zeta_{0}\right)$ on the magnetic surface. Figure 11 shows the eigenfrequency as a function of $\theta_{k}$, with other parameters the same as used in figure 3. The eigenfunctions of some of the modes are shown as insets. In both cases, the maximum growth rate is found at $\theta_{k}=0$, which means that the radial wavenumber vanishes at the matching point. Accordingly, the finite Larmor radius stabilization effect is weakest at this point. As $\theta_{k}$ increases, the frequencies and growth rate of these modes decrease and the corresponding eigenfunctions are shifted away from the symmetry point of the magnetic field and have a broader envelope. However, for large values of $\theta_{k}$ the strongly localized modes cease to exist. The results indicate that modes are strongly dependent on the orientation of the wave vector and thus propagate within the surface perpendicular to the field line. As was seen in figure 5, the growth rates and real frequencies in the non-circular case are smaller than in the circular tokamak except at the symmetry point, where the modes are most localized.

In tokamaks, as one moves in the poloidal direction the equilibrium quantities like magnetic field, normal and geodesic curvature and local magnetic shear change periodically. Therefore, the spectrum can also vary with the position of the matching point on the flux surface. This variation is calculated in figure 12 by moving the matching point $\theta_{0}$, and fixing $\zeta_{0}=0$, because of toroidal symmetry. As discussed in the work of Dewar and Glasser [15] the growth rate depends on the flux surface, the magnetic field line label and the radial wave number. In principle, one sets these three parameters and solves for the spectrum. However, the eigenmode spectrum associated with a specific set of these three parameters is beyond every attempt to obtain a solution in one go. This is not at all surprising as the field line in general is infinitely long, passing arbitrarily close to every point on a magnetic surface. Hence every attempt to solve for the full spectrum invariably picks up only part thereof. With the shooting method used in this paper, what we tend to pick up is the part of the spectrum localized around the matching points where the continuity of the eigenmodes is enforced. Hence, by moving the matching point around, one therefore recovers the complete spectrum. One can either do this by keeping the field line fixed and move the matching point on the field line or by moving from field line to field line. Formally, these two methods are not identical as in the latter case the three parameters are not held fixed. However, since every field line, on a non-rational surface, passes arbitrary close to every point on the surface, the two spectra obtained are identical in the limit of arbitrary fine resolution. That both these methods work in practice, and give the 

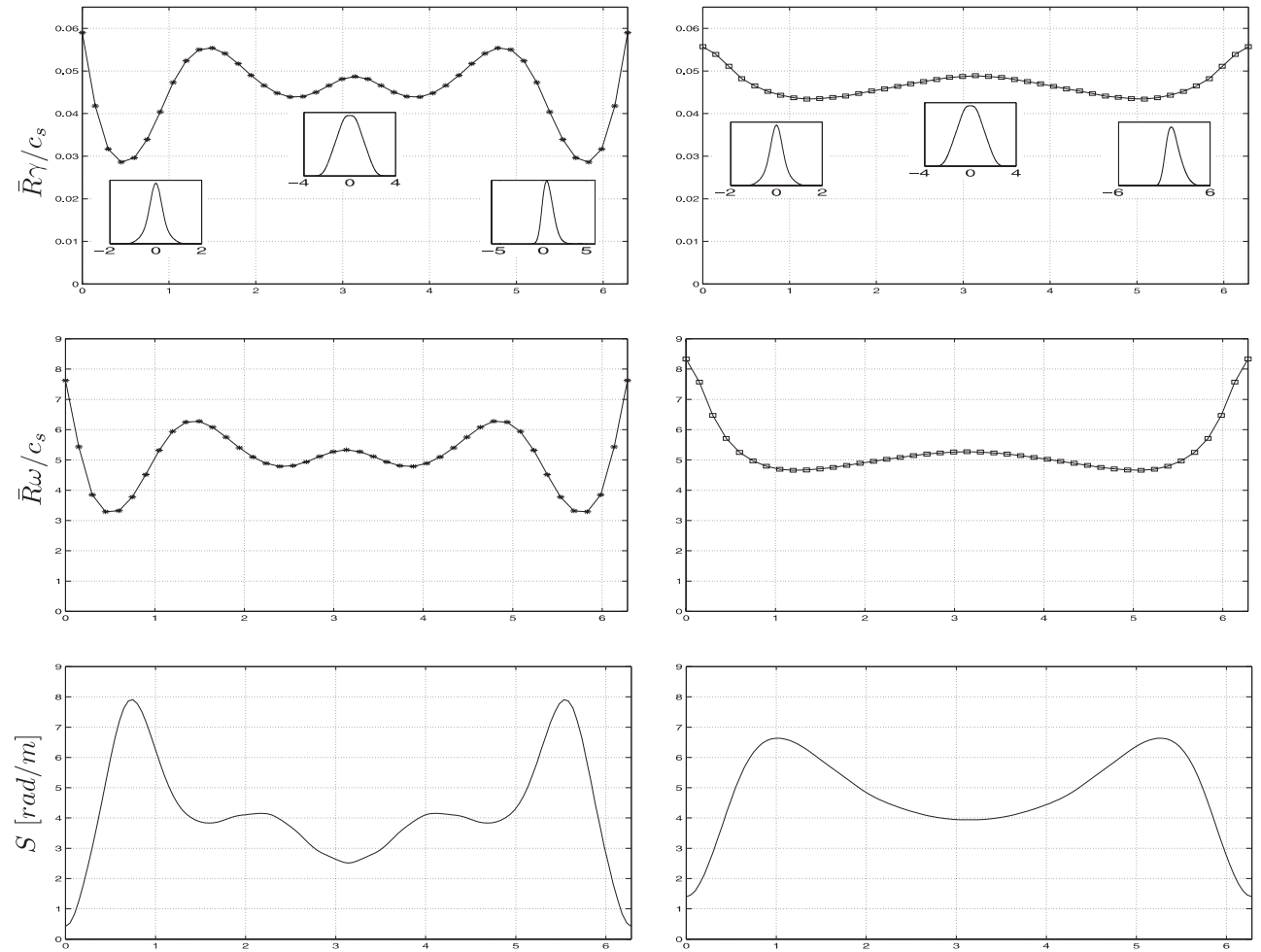

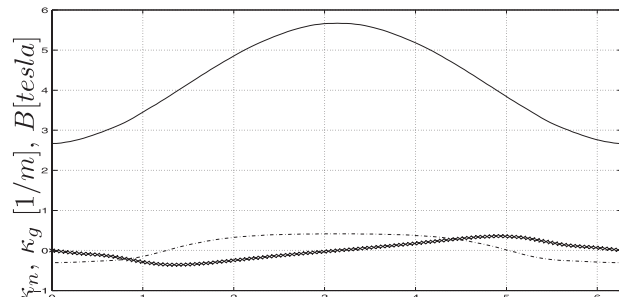

$\theta_{0}[\mathrm{rad}]$ along $\zeta_{0}=0$ line

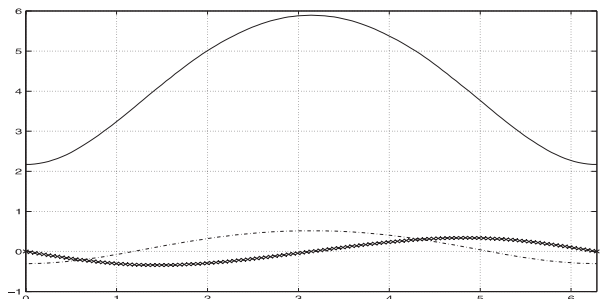

$\theta_{0}[\mathrm{rad}]$ along $\zeta_{0}=0$ line

Figure 12. Variation in the growth rate, frequency, local magnetic shear, $S$, change in field, $|\boldsymbol{B}|$, geodesic curvature, $\kappa_{\mathrm{g}}$, and the normal curvature, $\kappa_{\mathrm{n}}$, as a function of $\theta_{0}$ for the non-circular (left) and circular (right) cases. $|\boldsymbol{B}|$ is the solid curve (-), $\kappa_{\mathrm{g}}$ is the cross curve $(x)$ and $\kappa_{\mathrm{n}}$ is the dash-dotted curve ( $-\cdots)$ for $b=0.1$, and the other parameters are the same as in figure 7 .

same results, can easily be demonstrated numerically as well. What is done here is slightly more advanced. Since the growth rate has a maximum at $\theta_{k}=0, \theta_{k}$ is redefined in such a way that it sets the radial mode number to zero at each matching point and the most unstable modes on the magnetic surface are picked up. Figure 12 illustrates the variation of the growth rate, frequency, local magnetic shear, $|\boldsymbol{B}|$, normal curvature and geodesic curvature as a function of poloidal angle, $\theta_{0}$, for $\theta_{k}=0$, and the other parameters used are the same as in figure 11 . In both geometries, the growth rate is found to be strongly coupled to the local magnetic shear. As the magnitude of the local magnetic shear increases and unfavourable curvature decreases, the growth rate of the mode decreases as well. However, in the region of unfavourable curvature the modes are found to be localized, while the envelope of the eigenfunction becomes broader as 

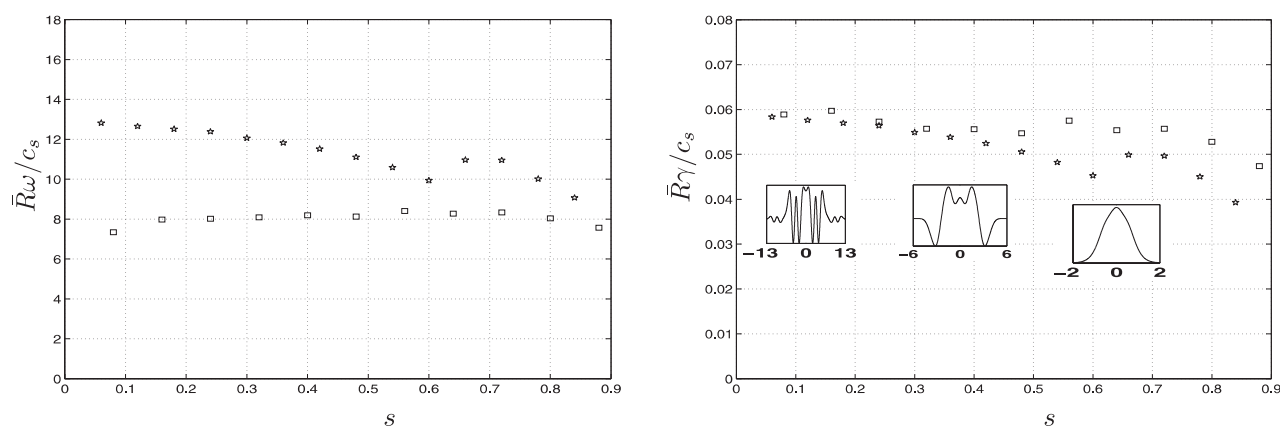

Figure 13. Normalized real frequency, $\bar{R} \omega / c_{s}$ (left), and growth rate, $\bar{R} \gamma / c_{s}$ (right), as a function of the surface label $s$ (squares representing the circular numerical and stars the circular analytical equilibria) for $\epsilon_{\mathrm{n}}=0.05, \theta_{k}=0, b=0.1$ and $\alpha=0$.
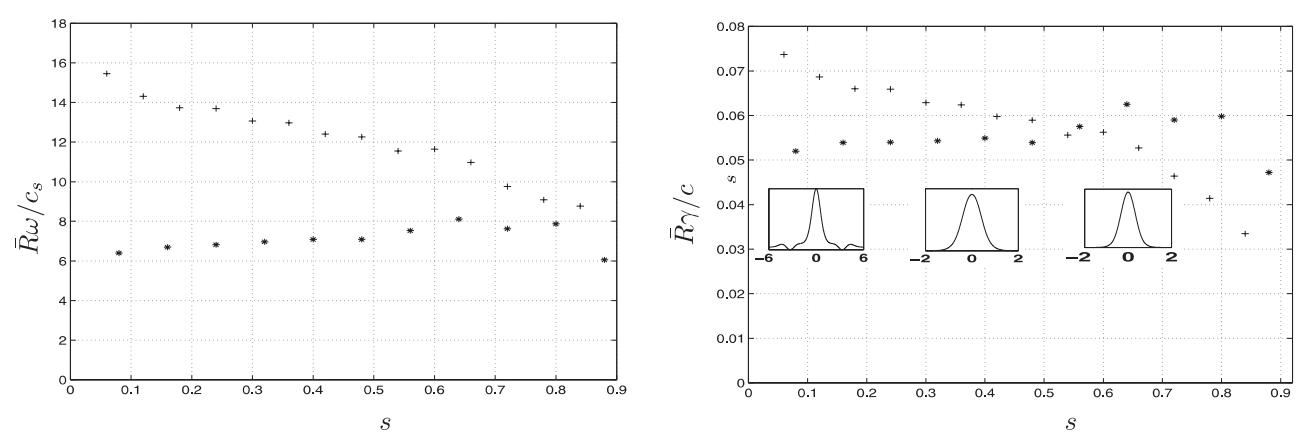

Figure 14. Normalized real frequency, $\bar{R} \omega / c_{s}$ (left), and growth rate, $\bar{R} \gamma / c_{s}$ (right), as a function of the surface label $s$ (asterisks representing the non-circular numerical and pluses the non-circular analytical equilibria) for $\epsilon_{\mathrm{n}}=0.05, \theta_{k}=0, b=0.1$ and $\alpha=0$.

it enters the favourable curvature region. Therefore, the highest growth rate and real frequency for both cases is found in regions where the local magnetic shear is small and normal curvature is unfavourable, that is at $\theta_{0}=0$.

A radial scan over the magnetic surface label $s$ is performed for circular tokamak equilibria in figure 13 and for non-circular tokamak equilibria in figure 14. In the process, $b=0.1$, $\theta_{0}=0, \epsilon_{\mathrm{n}}=0.05, \theta_{k}=0, \delta=0.01$ and $\alpha=0$ are kept fixed. It must be remarked here that $\epsilon_{\mathrm{n}}$ is not varying during this scan because our emphasis is on examining the effect of the geometry on the same mode rather than the effects of density profiles. In the case of numerical tokamak equilibria, we observed that, for small values of the radial normalized variable $s$, the growth rate is higher in the circular case than in the non-circular tokamak, whereas it has a lower growth rate for larger values of $s$. This is a consequence of the variation in the local magnetic shear strength that changes its magnitude from a lower value to a higher value as $s$ increases. This can be demonstrated by plotting the value of the local magnetic shear on the magnetic surface $s=0.8$ for the circular tokamak and the non-circular case. We note in figure 15 that the normal component of the curvature is found to be almost the same in both configurations, while the shear is considerably smaller for the circular tokamak and becomes even smaller for the simple infinite aspect ratio $\hat{s}-\alpha$ model equilibrium. The differences in the eigenvalues are found between the analytical and the numerical equilibria. This is due to decreasing the inverse aspect ratio $(\epsilon=\bar{R} / \bar{a}=2.96 / 1.675 \simeq 1.77)$ of analytical equilibria in order to compare and contrast its results with numerical circular and JET-like equilibria of same aspect ratio. This 

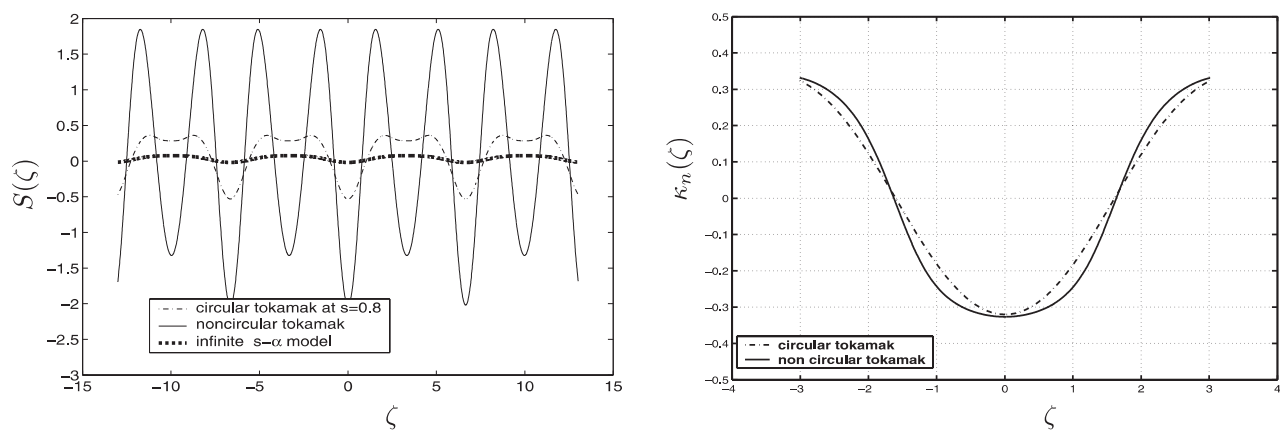

Figure 15. Variation of the shear, $S(\zeta)$ (left), and $\kappa_{\mathrm{n}}(\zeta)$ (right), along a magnetic field line with $\theta_{0}=0, \zeta_{0}=0$ on the magnetic surface $s=0.8$. The dash-dotted line represents the circular cross section tokamak, dotted line the simple circular infinite aspect ratio $\hat{s}-\alpha$ model equilibrium and solid line the JET.
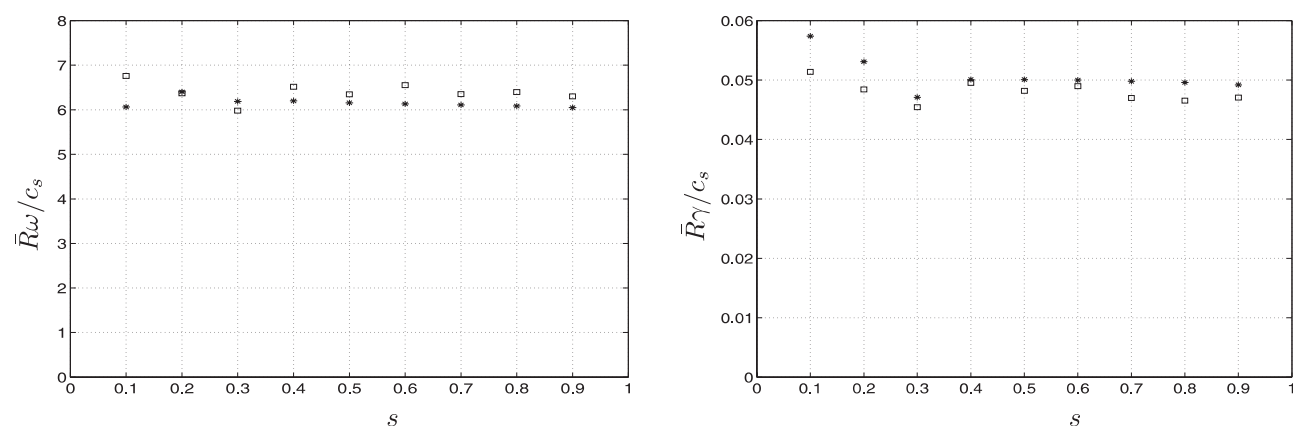

Figure 16. Normalized real frequency, $\bar{R} \omega / c_{s}$ (left), and growth rate, $\bar{R} \gamma / c_{s}$ (right), as a function of the surface label $s$ (squares representing the circular analytical aspect ratio 10 and stars the corresponding circular numerical equilibria); other parameters are the same as in figure 13 .

is checked by plotting the eigenvalue as a function of the normalized radial Boozer coordinate ' $s$ ' in figure 16 for the simple circular infinite aspect ratio $\hat{s}-\alpha$ model and is compared with the corresponding numerical equilibrium. They are found to be in good agreement. Moreover, the local magnetic shear plotted for the simple circular infinite aspect ratio $\hat{s}-\alpha$ model in figure 5 has smaller values than for the numerical equilibria. Hence large eigenvalues can be understood with the help of figure 12, where they are inversely proportional to the local magnetic shear. Finally, we have done an elongation scan which is presented in figure 17. This has been done using an analytical equilibrium and the results show destabilization for peaked density profiles. Maximum destabilization is found for $\kappa \leqslant 1.7$. As the equilibria become more elongated, the influence of $\kappa$ is reversed and a stabilization is observed for large values. A decrease in real frequency is also found due to elongation. This is because the magnetic drift frequency, $\Omega_{d}$, is expected to be reduced with increasing elongation.

\section{Summary}

A detailed comparison of the linear stability of electron drift modes is made between circular and non-circular tokamak geometries, which are also compared with $\hat{s}-\alpha$ model equilibria. A JET-like equilibrium is compared with a circular equilibrium with the same aspect ratio. The cold ion model used to describe these modes is derived from the fluid dynamics for ions 

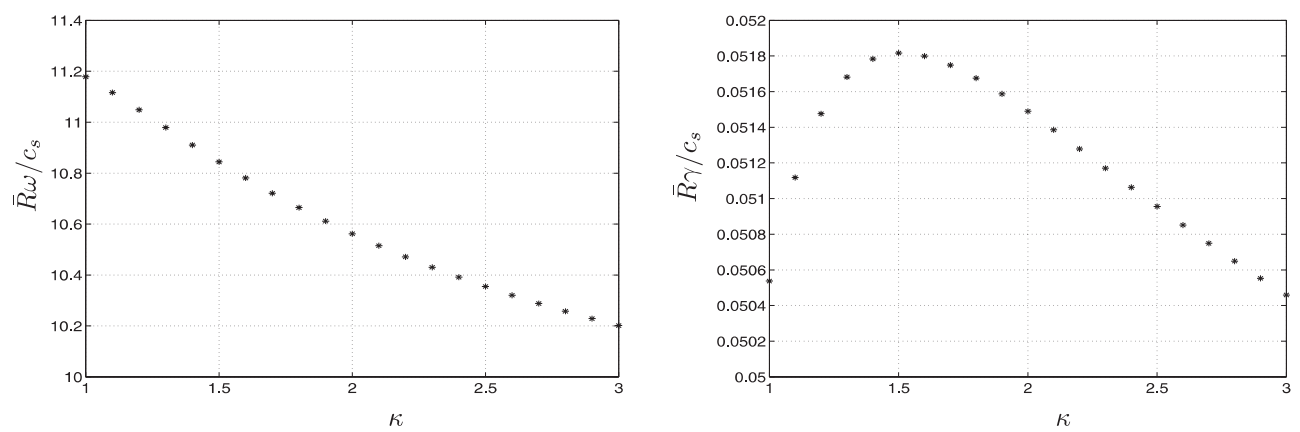

Figure 17. Normalized real frequency, $\bar{R} \omega / c_{s}$ (left), and growth rate, $\bar{R} \gamma / c_{s}$ (right), as a function of the elongation $(\kappa)$, using analytical equilibrium for $\epsilon_{\mathrm{n}}=0.05, \theta_{k}=0, b=0.1$ and $\alpha=0$.

in an electrostatic regime, whereas the electron response is assumed to be close to adiabatic. The eigenvalue problem for the drift wave equation is solved numerically using the ballooning mode formalism in fully three-dimensional tokamak geometries. Drift waves have been found everywhere on the flux surfaces. The high-frequency modes are strongly localized, while the low-frequency modes are weakly localized along the field lines. We have also found that the existence of the high-frequency modes, their localization along the field lines and their frequencies and growth rates are strongly dependent on the local shear of the magnetic field. The mode frequencies and growth rates are inversely proportional to the magnitude of the shear, while field line curvature has less influence on these drift modes. Modes are found to be more localized in a non-circular tokamak than in a circular equilibrium. As in stellarators, this is found to be due to the variation in the strength of the local magnetic shear, which localizes the modes along the field lines and constrains them. Shaping parameters, like elongation, do modify the perpendicular space variation and thereby the effective magnetic shear and one gets an enhanced convective damping due to magnetic shear. At the same time, however, the mode width is reduced and this tends to reduce the benifical effect on shear damping. This can be seen in figures 3, 4 and 17 of this paper. Therefore, in the non-circular low- $\beta$ equilibria considered, shaping turns out to be destabilizing. This has also been seen by Belli et al [21]. However, at high $\alpha$ (here $\alpha \approx-R_{0} / q^{2} \partial \beta / \partial \rho$ ), stabilizing effects have been observed due to the increase in triangularity at finite elongation. In summary, shaping is important for stability but can have both stabilizing and destabilizing influences. However, the stabilization due to compression is recovered for smaller values of $\epsilon_{\mathrm{n}}$ in the non-circular case than in the circular tokamak. A slight shift towards a shorter wavelength is found in the spectrum of the unstable modes for the non-circular case. This shift may tend to reduce the transport by reducing the correlation length in the plasma. The effect of non-circularity for peaked density profiles is found to be stabilizing in the inner radial region, whereas the effect is found destabilizing at the outer radial region. This is due to the variation of the local magnetic shear, which varies strongly in the non-circular case in comparison with the circular case. Finally, in the comparison of the numerical and the analytical equilibria results, differences are found. This is due to decreasing the inverse aspect ratio of analytical equilibria in order to compare and contrast its results with numerical circular and JET-like equilibria of same aspect ratio. This is checked by taking a simple circular infinite aspect ratio model and is compared with the corresponding numerical equilibrium. They are found to be in good agreement. Therefore, the analytical equilibria are found to be fine as long as the aspect ratio is large enough, but it has problems for smaller aspect ratios such as in JET. However, to validate this point, work on a more complete model including ion and electron temperature gradients and electron trapping to compare numerical equilibria 
with analytical equilibria including the effects of elongation, triangularity and Shafranov shift is in progress. Unstable drift modes are found both in favourable and unfavourable curvature regions. This is partly due to keeping the dissipative mechanism constant. While this is an artefact, it is also an advantage as it separates the driving mechanism from the effects of the magnetic configuration, which was the main purpose of this paper.

\section{Acknowledgments}

The authors are grateful to Drs M Nadeem, Pär Strand and M H Nasim for helpful discussions. This work was funded by the Euratom/VR association.

\section{References}

[1] Rudakov L I and Sagdeev R Z 1961 Sov. Phys. Dokl. 6415

[2] Coppi B, Rosenbluth M N and Sagdeev R Z 1967 Phys. Fluids 10582

[3] Coppi B and Pegoraro F 1977 Nucl. Fusion 17969

[4] Guzdar P N, Chen L, Tang W M and Rutherford P H 1983 Phys. Fluids 26673

[5] Jarmèn A, Andersson P and Weiland J 1987 Nucl. Fusion 27941

[6] Waltz R E and Miller R L 1999 Phys. Plasmas 64265

[7] Anderson J, Rafiq T, Nadeem M and Persson M 2002 Phys. Plasmas 91629

[8] Bernard L C and Moore R W 1979 Phys. Rev. Lett. 43965

[9] Charlton L A, Nelson D B and Dory R A 1980 Phys. Rev. Lett. 4524

[10] Hannum D et al 2001 Phys. Plasmas 8964

[11] Hirshman S P and Betancourt O 1991 J. Comput. Phys. 9699

[12] Miller R L et al 1998 Phys. Plasmas 5973

[13] Connor J W, Hastie R J and Taylor J B 1978 Phys. Rev. Lett. 40396

[14] D'haeseleer W D, Hitchon W N G, Callen J D and Shohet J L 1991 Flux Coordinates and Magnetic Field Structure (Berlin: Springer) chapter 6

[15] Dewar R L and Glasser A H 1983 Phys. Fluids 263038

[16] Boozer A H 1982 Phys. Fluids 25520

[17] Nührenberg J and Zille R 1987 Theory of Fusion Plasmas ed A Bondeson et al (Varenna: Editrice Compositori) p 3

[18] Greene J M and Chance M S 1981 Nucl. Fusion 21453

[19] Nadeem M, Rafiq T and Persson M 2001 Phys. Plasmas 91629

[20] Hua D D, Yu Y Q and Fowler T K 1992 Phys. Fluids 343216

[21] Belli E A, Hammett G W, Budny R V and Dorland W 2002 APS-DPP, Orlando

[22] Antonsen T M and Lane J B 1980 Phys. Fluids 231205

[23] Rafiq T, Anderson J, Nadeem M and Persson M 2001 Plasma Phys. Control. Fusion 431363

[24] Romanelli F 1989 Phys. Fluids B 11018 